\title{
Achados de Retossigmoidoscopias no Rastreamento de Câncer Colorretal em Pacientes Assintomáticos acima de 50 anos
}

\author{
Rectosigmoidoscopy for Colorectal câncer Screening in \\ Asymptomtic 50-Year-Old
}

\author{
CAROLINA VANNUCCI V. NOGUEIRADIOGENES ${ }^{1}$; RAPHAEL MARIANELLI ${ }^{1}$; RODRIGODE PÁDUA SAFATLE \\ SOARES $^{1}$; REGIS MIKAIL ABUD ${ }^{1}$; VINICIO FALLEIROS ${ }^{1}$; TEREZADE CARVALHO VILARIÑO ${ }^{1}$; MARISTELA GOMES \\ DE ALMEIDA $^{1}$; ANTONIOCARLOS BARAVIERA ${ }^{1}$
}

Hospital do Servidor Público Municipal de São Paulo ${ }^{1}$

\begin{abstract}
DIOGENES CVVN; MARIANELLI R; SOARES RPS; ABUD RM; FALLEIROS V; VILARIÑO TC; ALMEIDA MG; BARAVIERAAC. Achados de Retossigmoidoscopias no Rastreamento de Câncer Colorretal em Pacientes Assintomáticos Acima de 50 anos. Rev bras Coloproct, 2007;27(4): 403-407.

RESUMO: OBJETIVO: Analisar os achados do exame de retossigmoidoscopia no rastreamento de câncer colorretal em pacientes assintomáticos acima de 50 anos. MÉTODOS: Análise prospectiva de 208 pacientes assintomáticos acima de 50 anos e sem história familiar para câncer colorretal, que realizaram retossigmoidoscopia para rastreamento desta neoplasia durante o período de Janeiro de 2005 a Maio de 2006. Dos pacientes analisados, 64,5\% eram mulheres e a média de idade era de 60,24 anos (50-91 anos). RESULTADOS: Observamos que 4,8\% dos pacientes (n=10) apresentaram pólipos que variavam de 3 a $20 \mathrm{~mm}$ de tamanho. Todos os pólipos foram retirados e enviados para estudo anatomopatológico. Destes, 3,36\% $(n=7)$ foram hiperplásicos e 1,50\% $(n=3)$ eram adenomas, sendo dois adenoma tubular com atipias leves e um adenoma com atipias moderadas. Dos dez doentes que tinham pólipos ao exame, nove foram submetidos a videocolonoscopia e um abandonou o acompanhamento. Destes nove, sete pacientes apresentaram ausência de pólipos ao exame colonoscópico, e um apresentou dois pólipos adenomatosos em cólon direito. CONCLUSÕES: A retossigmoidoscopia é um exame acessível na maioria dos serviços que permite identificar pólipos potencialmente neoplásicos em pacientes assintomáticos.
\end{abstract}

Descritores: Sigmoidoscopia, câncer colorretal, rastreamento.

\section{INTRODUÇÃO}

O câncer colorretal é uma doença de grande prevalência em todo o mundo, ainda mantendo morbimortalidade significativa devido ao diagnóstico na maioria das vezes tardio. Nos Estados Unidos, levantamentos estatísticos o apontam como a segunda maior causa de morte por câncer (1). Apesar de vários estudos mostrarem a eficácia do rastreamento colorretal na prevenção de formas avançadas da doença pela detecção de lesões precoces e pólipos prémalignos, a adesão da população geral ao rastreamento é muito baixa: menos de $30 \%$ da população alvo foi submetida a exames de rastreamento colorretal nos Estados Unidos (2); no Brasil não há dados semelhantes, embora se acredite que a porcentagem seja ainda menor. Em contraste, $71 \%$ das mulheres americanas aderiram ao rastreamento para o câncer de mama e

Trabalho realizado no Serviço de Coloproctologia do Hospital do Servidor Público Municipal de São Paulo.

Recebido em 06/09/2007

Aceito para publicação em 23/10/2007 
Rev bras Coloproct

Outubro/Dezembro, 2007
Achados de Retossigmoidoscopias no Rastreamento de Câncer Colorretal em Pacientes Assintomáticos Acima de 50 anos

Carolina Vannucci V. Nogueira Diogenes e Cols. mais de $80 \%$ delas ao rastreamento de neoplasia de colo do útero através de Papanicolaou. A baixa adesão ao rastreamento colorretal deve-se a vários fatores, entre eles a resistência de médicos e pacientes, ao desconhecimento da existência deste rastreamento, ao alto custo de alguns dos exames envolvidos e as dificuldades logísticas do sistema de saúde (3)(30).

\section{OBJETIVO}

O objetivo deste trabalho foi analisar os achados do exame de retossigmoidoscopia no rastreamento de câncer colorretal em pacientes assintomáticos acima de 50 anos.

\section{PACIENTES E MÉTODOS}

Realizou-se uma análise prospectiva de 208 pacientes que realizaram retossigmoidoscopia para rastreamento de câncer colorretal no ambulatório de Coloproctologia do Hospital do Servidor Público Municipal de São Paulo, do período de Janeiro de 2005 a Maio de 2006..

Os critérios de inclusão para o estudo foram a idade acima de cinqüenta anos e ausência de sintomas sugestivos de neoplasia colorretal (dor abdominal, sangramento, alteração ou alternância do hábito intestinal, diarréia, mucorréia etc.). Os critérios de exclusão foram história familiar positiva para câncer colorretal, história prévia de neoplasia colorretal ou em

Tabela 1 - Distribuição dos pacientes com retossigmoidoscopia normal e alterada.

\begin{tabular}{lrc}
\hline & Exame normal & Exame alterado \\
\hline Homens & $71(34,13 \%)$ & $03(1,44 \%)$ \\
Mulheres & $127(61,05 \%)$ & $07(3,36 \%)$ \\
Total & $198(94,73 \%)$ & $10(5,26 \%)$ \\
\hline
\end{tabular}

outros sítios, presença de sintomas sugestivos de neoplasia colorretal e recusa de participar do estudo.

O exame de retossigmoidoscopia foi realizado ambulatorialmente, com preparo prévio do reto e cólon distal com enema de fosfato de sódio. O aparelho utilizado foi o retossigmoidoscópio rígido descartável de $25 \mathrm{~cm}$ de comprimento, acoplado a fonte de luz. Nos casos onde encontramos alterações neoplásicas (pólipos), as lesões foram retiradas e enviadas para estudo anatomopatológico e os pacientes foram submetidos a investigação colônica por videocolonoscopia.

\section{RESULTADOS}

No total de 208 pacientes analisados, $35,5 \%$ eram homens e $64,5 \%$ eram mulheres. A média de idade encontrada foi de 60,24 anos, onde a idade máxima foi de 91 anos (tabela 1).

No grupo, $94,73 \%$ dos exames foram considerados normais e 5,26\% apresentaram alterações neoplásicas (pólipos) onde $70 \%$ (3,36\% do grupo total) eram hiperplásicos e $30 \%$ (1,5\%do total) eram adenomas tubulares com variados graus de atipia (tabela 2). Estes pacientes foram submetidos a videocolonoscopia, onde dos pacientes com pólipos hiperplásicos um apresentou pólipos adenomatosos em cólon direito, cinco apresentaram exame normal e um não realizou o exame; e dos pacientes com pólipos adenomatosos um apresentou exame normal, um apresentou angiodisplasia em sigmóide sem lesões neoplásicas e um não realizou o exame (tabela 3).

Tabela 2 - Resultados do estudo anatomopatológico dos pólipos encontrados:

\begin{tabular}{|c|c|}
\hline Tipo histológico & Tamanho \\
\hline Tubulares com atipias leves $(n=2)$ & $20 \mathrm{~mm}$ e $6 \mathrm{~mm}$ \\
\hline Tubulares sem atipias $(\mathrm{n}=1)$ & $5 \mathrm{~mm}$ \\
\hline Hiperplásicos (n=7) & $8 \mathrm{~mm}$ \\
\hline
\end{tabular}

Tabela 3 - Resultados do estudo colonoscópico nos pacientes com pólipos na retossigmoidoscopia.

$\begin{array}{cc}\begin{array}{c}\text { Pólipos hiperplásicos } \\ \left(n^{\circ} \text { Pacientes }\right)\end{array} & \begin{array}{c}\text { Adenomas } \\ \left(n^{\circ} \text { Pacientes }\right)\end{array}\end{array}$

\begin{tabular}{lll}
\hline Sem pólipos & 5 & 2 \\
Pólipos proximais & 1 & 0 \\
Não realizou & 1 & 1 \\
\hline
\end{tabular}


Achados de Retossigmoidoscopias no Rastreamento de Câncer Colorretal em Pacientes Assintomáticos Acima de 50 anos Carolina Vannucci V. Nogueira Diogenes e Cols.

\section{DISCUSSÃO}

Apesar das recomendações para o rastreamento ainda variarem nos detalhes, é senso comum à importância do mesmo na prevencão das neoplasias colorretais. Essas variações representam várias perspectivas em relação às metas desejadas no rastreamento do câncer colorretal; as recomendações mais agressivas refletem a intenção de que nenhum caso de câncer seja omitido, enquanto as recomendações mais baseadas em estudos populacionais tentam disponibilizar o rastreamento para o maior número de pacientes possível. Se a primeira visão for favorecida, a colonoscopia ganha importância como exame de rastreamento mais adequado, porém se o objetivo for a redução de taxas populacionais de morte por câncer colorretal deve se favorecer exames com maior alcance populacional pela maior disponibilidade e menor custo (34).

O rastreamento de pacientes com baixo risco para desenvolvimento de câncer colorretal envolve a pesquisa de sangue oculto nas fezes anualmente, com sigmoidoscopia flexível a cada cinco anos ou retossigmoidoscopia rígida a cada dois anos, a partir dos cinquienta anos. Os pacientes com alto risco para desenvolvimento de câncer colorretal são rastreados com colonoscopia, a partir dos quarenta anos. A escolha dos exames empregados em pacientes de baixo risco envolve muitas considerações, como os valores preditivos de cada teste, custo, facilidade de emprego e disponibilidade (34)(35).

A pesquisa de sangue oculto nas fezes é o exame para rastreamento mais realizado nos Estados Unidos, com relação comprovada entre a realização do exame e a redução da mortalidade por câncer colorretal. A análise deve incluir três amostras seriadas de acordo com a literatura, pois a análise de uma amostra apenas aumenta a incidência de falsos positivos ou negativos (4)(5)(6). Sua principal vantagem é a acessibilidade do exame, porém apresenta uma elevada taxa de resultados falso positivos e negativos. Mais recentemente, novos métodos de análise imunohistoquímica foram adaptados à pesquisa de sangue oculto nas fezes, aumentando a acurácia do exame para rastreamento de neoplasia colorretal sem a necessidade de restrição dietética. A aplicação deste novo teste para rastreamento permanece em estudo, com resultados promissores (36).
A colonoscopia é um exame que acessa todo o cólon, sendo considerado o exame mais acurado para a pesquisa de pólipos e neoplasias colônicas. Requer um endoscopista treinado para sua execução e exige preparo de cólon elaborado e sedação do pacientes. Se comparado com a sigmoidoscopia, a colonoscopia tem maior taxa de perfuração colônica ( 2 a cada 1000 procedimentos, ao que a retossigmoidoscopia apresenta 1 perfuração a cada 10000 exames (9)), maior taxa de complicações relacionadas à sedação e maior custo. A colonoscopia, atualmente, vem recebendo maior atenção por ser o teste mais preciso para detecção de lesões precoces ou pré-malignas, e possibilitar a ressecção terapêutica das lesões a um só tempo. Porém, é um exame caro, invasivo e pouco disponível, envolvendo dificuldades logísticas que inviabilizam sua aplicação em larga escala (9). Assim, de acordo com os atuais consensos, é um exame reservado para pacientes com alto risco de desenvolvimento de câncer colorretal.

A retossigmoidoscopia rígida é um exame de baixo custo e largamente disponível no sistema de saúde, que não requer sedação para sua realização e não exige preparo de todo o cólon, sendo possível sua realização ambulatorial por médicos ou profissionais de saúde adequadamente treinados com índices de complicações muito baixos. Apresenta como desvantagem principal a visão limitada ao cólon distal e reto, assim as lesões proximais ao aparelho não estariam ao seu alcance direto, falhando em diagnosticar metade das lesões proximais do cólon (13)(14).

Na casuística estudada neste trabalho, 5,26\% dos pacientes apresentaram pólipos ao exame retossigmoidoscópico, o que está abaixo dos achados de literatura, onde pelo menos $25 \%$ da população em geral apresentaria pelo menos um pólipo ao exame. Porém, menos da metade desses pacientes apresentariam adenomas à biópsia, sendo os restantes pólipos hiperplásicos (17). Todos os pacientes com achado de pólipos à retossigmoidoscopia foram submetidos à videocolonoscopia, onde em 01 paciente encontrou-se pólipos em segmentos proximais. Isso mostra a boa capacidade de rastreamento de pacientes assintomáticos por retossigmoidoscopia, considerando a indicação de colonoscopia para qualquer lesão encontrada no exame.

É importante ressaltar que o paciente que apresentou pólipos adenomatosos à colonoscopia apresentava pólipos hiperplásicos no exame de 
Rev bras Coloproct Outubro/Dezembro, 2007
Achados de Retossigmoidoscopias no Rastreamento de Câncer Colorretal em Pacientes Assintomáticos Acima de 50 anos Carolina Vannucci V. Nogueira Diogenes e Cols. retossigmoidoscopia, mostrando que a indicação de estudo completo do cólon não deve se restringir a achados de pólipos adenomatosos.

A indicação de estudo colônico completo ao achado de lesões na retossigmoidoscopia permanece controversa. A sensibilidade do exame para detectar lesões proximais depende do limiar de indicação de colonoscopia nos exames alterados; por exemplo, se a presença de qualquer pólipo é usado como limiar, a sensibilidade da retossigmoidoscopia para detecção de lesões proximais aumenta de $50 \%$ para $70 \%$. Estatísticas americanas mostram que a taxa de mortalidade por câncer colorretal é reduzida em até $60 \%$ pelo achado de uma lesão por sigmoidoscopia, o que aplica uma porcentagem de $30 \%$ com relação a todo o có$\operatorname{lon}(17)(21)(33)$.

Apesar da impossibilidade da retossigmoidoscopia detectar lesões colônicas proximais ter recebido muita atenção em editoriais(26) e da imprensa leiga, essa constatação não surpreendeu a especialistas e organizações profissionais, pois a sigmoidoscopia não foi projetada para ser um método particularmente sensível de detecção de lesões proximais. Os estudos que comparam os dois métodos não levam em conta a segurança, custo e disponibilidade de ambos os testes.

\section{CONCLUSÕES}

Embora não visualize todo o cólon, a retossigmoidoscopia é um exame acessível à maioria dos serviços, de fácil execução e mais seguro e barato que a colonoscopia, permitindo a identificação de lesões potencialmente neoplásicas em pacientes assintomáticos. Contudo, deve ser combinada a outros exames de rastreamento, aumentando sua sensibilidade conforme a associação ao estudo endoscópico completo do cólon.

ABSTRACT: PURPOSE: Analyze the findings of rigid retossigmoidoscopy in asymptomatic patients above 50 years old. METHOD: Prospective analysis of $\mathbf{2 0 8}$ asymptomatic patients above $\mathbf{5 0}$ years and without familiar history of colorectal cancer which were submitted to rigid retossigmoidoscopy for colorectal cancer screening on a period of 14 months. RESULTS: $4.8 \%$ of the studied patients had polyps which size varied from $3 \mathrm{~mm}$ to $20 \mathrm{~mm}$. All polyps were removed and submitted to pathological examination, where $3.36 \%(n=7)$ were hyperplastic polyps and $1.50 \%(n=3)$ were adenomas. The patients with polyps on sigmoidoscopic exam then were submitted to videocolonoscopic investigation; of these, seven patients had no other polyps on examination and one had two polyps on right colon. CONCLUSION: The rigid sigmoidoscopy is an accessible and safe tool for colorectal cancer screening, allowing identifying premalignant lesions in asymptomatic patients.

Key words: Colorectal cancer; rectosigmosdoscopy.

\section{REFERÊNCIAS}

1. Greenlee RT, Hill-Harmon MB, Murray T, Thun M. Cancer statistics, 2001. CA Cancer J Clin 2001;51:15-36. [Erratum, CA Cancer J Clin 2001;51:144.]

2. Screening for colorectal cancer - United States, 1997. MMWR Morb Mortal Wkly Rep 1999;48:116-21.

3. Vernon SW. Participation in colorectal cancer screening: a review. J Natl Cancer Inst 1997;89:1406-22.

4. Mandel JS, Bond JH, Church TR, et al. Reducing mortality from colorectal cancer by screening for fecal occult blood. $\mathrm{N}$ Engl J Med 1993;328:1365-71. [Erratum, N Engl J Med 1993;329:672.]

5. Kronborg O, Fenger C, Olsen J, Jorgensen OD, Sondergaard O. Randomised study of screening for colorectal cancer with faecal-occult-blood test. Lancet 1996;348:1467-71.

6. Hardcastle JD, Chamberlain JO, Robinson MHE, et al. Randomised controlled trial of faecal-occult-blood screening for colorectal cancer. Lancet 1996;348:1472-7.
7. Ransohoff DF, Lang CA. Screening for colorectal cancer with the fecal occult blood test: a background paper. Ann Intern Med 1997;126:811-22.

8. Lang CA, Ransohoff DF. What can we conclude from the randomized controlled trials of fecal occult blood test screening? Eur J Gastroenterol Hepatol 1998;10:199-204.

9. Pignone MP, Rich M, Teutsch SM, Berg AO, Lohr KN. Screening for colorectal cancer in adults. Systematic evidence review no. 7. Rockville, Md.: Agency for Healthcare Research and Quality (in press). (AHRQ publication no. 02-S003.)

10. Selby JV, Friedman GD, Quesenberry CP Jr, Weiss NS. A case-control study of screening sigmoidoscopy and mortality from colorectal cancer. N Engl J Med 1992;326:653-7.

11. Levin TR, Conell C, Shapiro JA, Chazan SG, Nadel M, Selby JV. Complications of screening sigmoidoscopy. Gastroenterology 2001;120:Suppl:A-65. abstract.

12. Ashley OS, Nadel M, Ransohoff DF. Achieving quality in flexible sigmoidoscopy screening for colorectal cancer. Am J Med 2001;111:643-53. 
13. Lieberman DA, Weiss DG, Bond JH, Ahnen DJ, Garewal H, Chejfec G. Use of colonoscopy to screen asymptomatic adults for colorectal cancer. N Engl J Med 2000;343:162-8. [Erratum, N Engl J Med 2000;343:1204.]

14. Imperiale TF, Wagner DR, Lin CY, Larkin GN, Rogge JD, Ransohoff DF. Risk of advanced proximal neoplasms in asymptomatic adults according to the distal colorectal findings. N Engl J Med 2000;343:169-74.

15. Lieberman DA, Weiss DG. One-time screening for colorectal cancer with combined fecal occult-blood testing and examination of the distal colon. N Engl J Med 2001;345:55560.

16. Kramer BS, Gohagan J, Prorok PC, Smart C. A National Cancer Institute sponsored screening trial for prostatic, lung, colorectal, and ovarian cancers. Cancer 1993;71:Suppl:58993.

17. Atkin WS, Edwards R, Wardle J, Northover JM, Cuzick J. UK Flexible Sigmoidoscopy Screening trial: compliance, yield and adverse effects. Gastroenterology 2000;118:Suppl:A187. abstract.

18. Rex DK, Johnson DA, Lieberman DA, Burt RW, Sonnenberg A. Colorectal cancer prevention 2000: screening recommendations of the American College of Gastroenterology. Am J Gastroenterol 2000;95:868-77.

19. Screening for colorectal cancer. In: Preventive Services Task Force. Guide to clinical preventive services. 2nd ed. Baltimore: Williams \&?a?m?p?; Wilkins, 1996:89-103.

20. Winawer SJ, Fletcher RH, Miller L, et al. Colorectal cancer screening: clinical guidelines and rationale. Gastroenterology 1997;112:594-642. [Errata, Gastroenterology 1997;112:1060, 1998; 114:625.]

21. Smith RA, von Eschenbach AC, Wender R, et al. American Cancer Society guidelines for the early detection of cancer: update of early detection guidelines for prostate, colorectal, and endometrial cancers. Also: update 2001 - testing for early lung cancer detection. CA Cancer J Clin 2001;51:38-75. [Erratum, CA Cancer J Clin 2001;51:150.]

22. Saitoh Y, Waxman I, West AB, et al. Prevalence and distinctive biologic features of flat colorectal adenomas in a North American population. Gastroenterology 2001;120:1657-65.

23. Winawer SJ, Stewart ET, Zauber AG, et al. A comparison of colonoscopy and double-contrast barium enema for surveillance after polypectomy. N Engl J Med 2000;342:1766-72.

24. Herrinton LJ, Selby JV, Friedman GD, Quesenberry CP, Weiss NS. Case-control study of digital-rectal screening in relation to mortality from cancer of the distal rectum. Am J Epidemiol 1995;142:961-4.
25. Fenlon HM, Nunes DP, Schroy PC III, Barish MA, Clarke PD, Ferrucci JT. A comparison of virtual and conventional colonoscopy for the detection of colorectal polyps. N Engl J Med 1999;341:1496-503. [Erratum, N Engl J Med 2000;342:524.]

26. Podolsky DK. Going the distance: the case for true colorectalcancer screening. N Engl J Med 2000;343:207-8.

27. Frazier AL, Colditz GA, Fuchs CS, Kuntz KM. Costeffectiveness of screening for colorectal cancer in the general population. JAMA 2000;284:1954-61.

28. Sonnenberg A, Delco F, Inadomi JM. Cost-effectiveness of colonoscopy in screening for colorectal cancer. Ann Intern Med 2000;133:573-84.

29. Wagner JL, Behney CJ, Tunis SR, Ching A. Cost-effectiveness of colorectal cancer screening in average-risk adults. Washington, D.C.: Office of Technology Assessment, 1995. (Report no. BP-H-146.)

30. Redelmeier DA, Shafir E. Medical decision making in situations that offer multiple alternatives. JAMA 1995;273:302-5.

31. Imperiale TF, Wagner DR, Lin CY, Larkin GN, Rogge JD, Ransohoff DF. A new clinical index to stratify risk for advanced proximal neoplasia in asymptomatic adults. Gastroenterology 2001;120:Suppl:A-15. abstract.

32. Mandel JS, Church TR, Ederer F, Bond JH. Colorectal cancer mortality: effectiveness of biennial screening for fecal occult blood. J Natl Cancer Inst 1999;91:434-7.

33. Byers T, Levin B, Rothenberger D, Dodd GD, Smith RA. American Cancer Society guidelines for screening and surveillance for early detection of colorectal polyps and cancer: update 1997. CA Cancer J Clin 1997;47:154-60.

34. Woolf SH. The best screening test for colorectal cancer - a personal choice. N Engl J Med 2000;343:1641-3.

35. Lieberman T. Matching colon tests to patients, not costs. Los Angeles Times. March 19, 2001:S1, S7.

36. Levi Z; Rosen P; Hazazi R; Vilkin A; Walked A; Maoz E; Birkenfeld $S$ et al. A quantitative immunochemical fecal occult blood test for colorectal neoplasia. Ann Int Med 2007; 1464;224-225.

\section{Endereço para correspondência:}

MARISTELA GOMES DE ALMEIDA

Rua Dr. Bacelar, 173 - Conj103

Vila Clementino - São Paulo/SP.

CEP: 04026-000

E-mail:dramgalmeida@uol.com.br 